\title{
Draxin Inhibits Axonal Outgrowth through the Netrin Receptor DCC
}

\author{
Giasuddin Ahmed, ${ }^{1,2,3}$ Yohei Shinmyo, ${ }^{1,2}$ Kunimasa Ohta, ${ }^{1}$ Shahidul M. Islam, ${ }^{1,2}$ Mahmud Hossain, ${ }^{1,3}$ \\ Iftekhar Bin Naser, ${ }^{1,2,3}$ M. Asrafuzzaman Riyadh, ${ }^{1,3}$ Yuhong Su, ${ }^{1,2,3}$ Sanbing Zhang, ${ }^{1,2,3}$ Marc Tessier-Lavigne, ${ }^{4}$ \\ and Hideaki Tanaka ${ }^{1,2,3}$ \\ ${ }^{1}$ Division of Developmental Neurobiology, Graduate School of Medical Sciences, Kumamoto University, Kumamoto 860-8556, Japan, 221st Century Center \\ of Excellence Cell Fate Regulation Research and Education Unit, and ${ }^{3}$ Global Center of Excellence Cell Fate Regulation Research and Education \\ Unit, Kumamoto University, Kumamoto 860-0811, Japan, and ${ }^{4}$ Laboratory of Brain Development and Repair, Rockefeller University, New York, \\ New York 10065
}

Draxin, a recently identified axon guidance protein, is essential for the formation of forebrain commissures, and can mediate repulsion of netrin-stimulated spinal commissural axons. Here, we report that draxin binds multiple netrin receptors: DCC (deleted in colorectal cancer), Neogenin, UNC5s (H1, H2, H3), and DSCAM (Down's syndrome cell adhesion molecule). Since draxin and Dcc knockouts showed similar phenotype in forebrain commissures formation, we show here the functional importance of draxin/DCC interaction. Draxin interacts with subnanomolar affinity to the netrin receptor DCC, in a region of DCC distinct from its netrin-binding domain. In vitro, neurite outgrowth from cortical and olfactory bulb explants of $D c c$ knock-out mice is significantly less inhibited by draxin, when compared with neurites from explants of wild-type mice. Furthermore, in comparison with wild-type mice, the growth cone collapse in response to draxin is largely abolished in Dcc-deficient cortical neurons. In vivo, double heteros of draxin/Dcc mice show markedly higher frequency of complete agenesis of corpus callosum than either of the single hetero. These results identify DCC as a convergent receptor for netrin and draxin in axon growth and guidance.

\section{Introduction}

The function of the nervous system depends on the establishment of precise and intricate neuronal connections. The combinatorial effects of attractive and repulsive guidance cues, present in the extracellular environment, direct axons to grow toward their intermediate targets and thus play a crucial role in forming proper connections between neurons. Four conserved families of axon guidance cues, the netrin, semaphorin, ephrin, and Slit proteins,

Received Feb. 22, 2011; revised June 12, 2011; accepted June 29, 2011.

Author contributions: G.A. and H.T. designed research; G.A., Y. Shinmyo, K.O., S.M.I., M.H., I.B.N., M.A.R., Y. Su, and S.Z. performed research; G.A., Y. Shinmyo, M.T.-L., and H.T. analyzed data; G.A., M.T.-L., and H.T. wrote the paper.

This work was supported by grants-in-aid from the Ministry of Education, Science, Sports, and Culture of Japan (MEXT); by the 21st Century Center of Excellence (COE) Program and by the Global COE Program (Cell Fate Regulation Research and Education Unit), MEXT, Japan; and by Takeda Science Foundation. Deletion constructs of Dcc and mouse DSCAM-myc were kind gifts from Drs. D. J. Leahy (The Johns Hopkins University, Baltimore, MD) and E. Stein (Yale University, New Haven, (T). We are very grateful to Drs. Hirohide Takebayashi and Keisuke Watanabe for providing the mice and primers; and to Katsuhiko 0 no and Toshihide Yamashita for providing the vectors encoding rat Dcc, VSVG-tagged human Neogenin, and HA-tagged rat UNC5H2. We are thankful to Drs. Tatsumi Hirata and Takahiko Kawasaki for allowing us to screen their cDNA library.

The authors declare no competing financial interests.

Correspondence should be addressed to Hideaki Tanaka, Division of Developmental Neurobiology, Graduate School of Medical Sciences, Kumamoto University, 1-1-1 Honjo, Kumamoto 860-8556, Japan. E-mail: hitanaka@kumamoto-u.ac.jp.

S. M. Islam's present address: Department of Molecular, Cellular, and Developmental Biology, Yale University, 266 Whitney Avenue, New Haven, С 06520.

Y. Su's present address: Department of Human Anatomy, Hebei Medical University, Shijiazhuang 050017, People's Republic of China.

S. Zhang's present address: Department of Orthopedics, The Third Hospital of Shijiazhuang City, Shijiazhuang 050011, People's Republic of China.

DOI:10.1523/JNEUROSCI.0943-11.2011

Copyright $\odot 2011$ the authors $\quad 0270-6474 / 11 / 3114018-06 \$ 15.00 / 0$ mediate their guidance effects via receptors of the DCC (deleted in colorectal cancer) or UNC5, neuropilin/plexin, Eph, and Robo families, respectively. Although these proteins, as well as morphogens such as Shh (sonic hedgehog), Wnts, and BMPs, have been found to regulate a wide variety of guidance decisions (for review, see TessierLavigne and Goodman, 1996; Dickson, 2002; Zou and Lyuksyutova, 2007), it is expected that others await identification and will help address the immense complexity of the nervous system.

We recently reported the discovery of a novel axon guidance molecule, draxin, which shares no sequence homology with other known guidance molecules (Islam et al., 2009). In vitro, draxin can repel spinal commissural axons whose outgrowth is stimulated by netrin-1; in vivo, genetic deletion of draxin results in mild guidance defects of those axons and a dramatic loss of all forebrain commissures (Islam et al., 2009). Identification of the draxin receptor is important in elucidating the precise mechanism through which it functions. Here, we report that draxin binds multiple netrin-1 receptors and that its high-affinity interaction with DCC is required for draxin's inhibitory effect on the cortical and olfactory bulb (OB) neurite outgrowth. The frequency of severity in corpus callosum (CC) malformation in draxin/Dcc double-hetero mice is more obvious than that in either of the single-hetero mice. This study identifies DCC as a functional receptor or receptor component for draxin.

\section{Materials and Methods}

Plasmids. Rat Dcc (Keino-Masu et al., 1996) was used in draxin binding experiments. Full-length cDNAs encoding rat Ncam, human NeogeninVSVG, UNC5H1-myc, UNC5H2-HA, UNC5H3-myc, mouse Dscam 

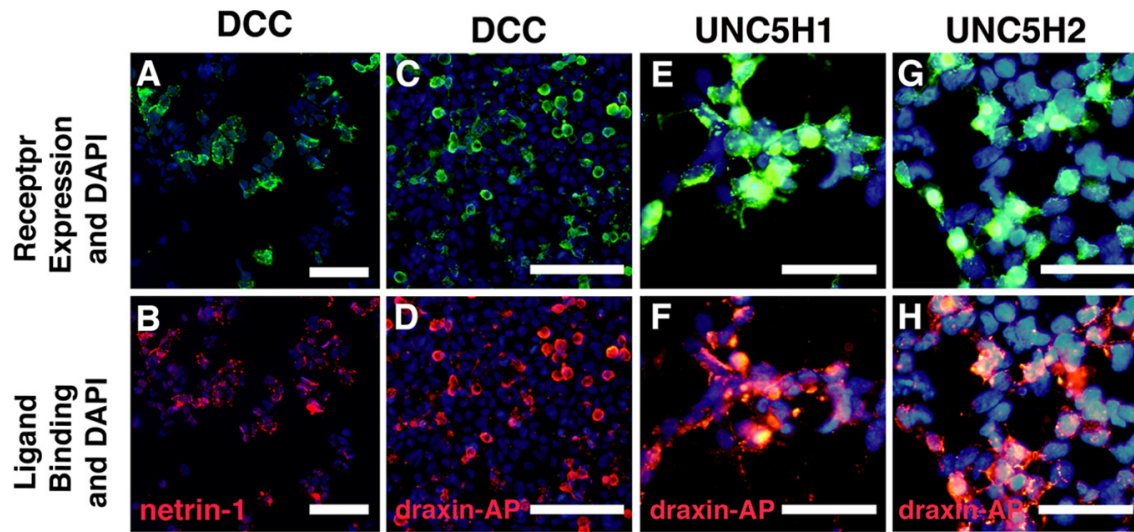

UNC5H3
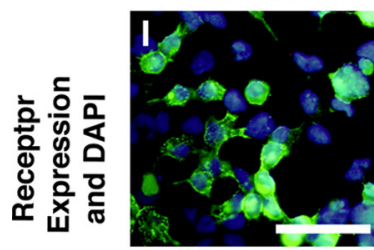

DSCAM

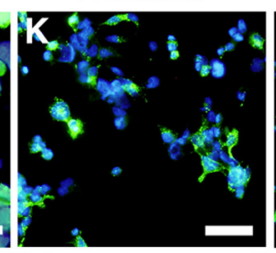

NCAM
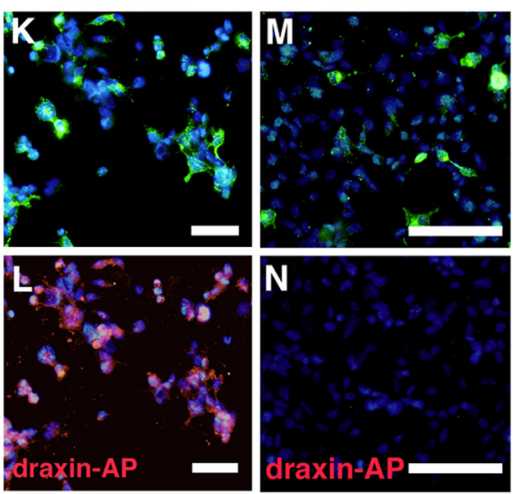

Figure 1. Draxin binds netrin-1 receptors. The 293 cells were transiently transfected with receptor CDNAs: $A-D, D c c ; E, F$, UNC5H1; $\boldsymbol{G}, \boldsymbol{H}$, UNCSH2; $\boldsymbol{I}, \boldsymbol{J}$, UNCSH3; $\boldsymbol{K}, \boldsymbol{L}$, Dscam; $\boldsymbol{M}, \boldsymbol{N}$, Nscam; $\boldsymbol{O}, \boldsymbol{P}$, Neogenin. After $40 \mathrm{~h}$ of post-transfection, cells were treated either with netrin-1 protein $(\boldsymbol{A}, \boldsymbol{B})$ or with draxin-AP-conditioned medium $(\boldsymbol{C}-L, N, P)$ and double immunostaining was performed using antibodies against respective receptors $(A, C, E, G, I, K)$ and against bound netrin-1 $(B)$ or draxin $(D, F, H, J, L)$. Bound draxin and receptor expression were merged in a manner similar to netrin- 1 binding $(\boldsymbol{B})$ and DCC expression $(\boldsymbol{A})$. In cases of NCAM-draxin $(\boldsymbol{M}, \boldsymbol{N})$ and Neogenin-draxin $(\boldsymbol{O}, \boldsymbol{P})$ binding assay, staining of NCAM or Neogenin protein and bound draxin were done separately, since all anti-NCAM, anti-Neogenin, and anti-draxin antibodies were rabbit polyclonal antibodies. A similar extent of draxin binding $(\boldsymbol{P})$ and Neogenin expression $(\boldsymbol{O})$ was observed, while draxin did not bind to the NCAM-transfected cells $(\boldsymbol{N})$. Scale bars: $A-D, M, N, 100 \mu \mathrm{m} ; E-L, O, P, 50 \mu \mathrm{m}$.

(Down's syndrome cell adhesion molecule)-myc, and extracellular domain of rat Robo1-Fc were a kind gift from Drs. K. Itoh (Tokushima Bunri University, Tokushima, Japan), E. Fearon (University of Michigan, Ann Arbor, MI), P. Mehlen (CNRS, Lyon, France), E. Stein (Yale University, New Haven, CT), and F. Murakami (Osaka University, Osaka, Japan), respectively. The extracellular domain of rat Dcc was subcloned into pEF-Myc-His vector (Invitrogen). Different types of human DCC cDNA fragments encoding DCC-ecto- $h \mathrm{GH}$-His, DCC-Fn(1-6)- $h \mathrm{GH}$ His, and DCC-IgG (1-4)-hGH-His (Geisbrecht et al., 2003) were a generous gift from Dr. D. J. Leahy (The Johns Hopkins University, Baltimore, MD).

Antibodies. Mouse monoclonal and rabbit polyclonal anti-chick draxin antibodies (Islam et al., 2009) were used for Western blot and immunoprecipitations. For the detection of netrin-1-His, NCAM, DCC, UNC5 (H1, H3), UNC5H2, Robo-ecto-Fc, fragmented DCC-hGH, and Neogenin, rabbit polyclonal anti-His (Santa Cruz Biotechnology), rabbit polyclonal antichicken NCAM (in house), mouse monoclonal anti-DCC (Calbiochem), anti-myc (9E10, DSHB), anti-HA (Sigma), rabbit polyclonal anti-human Fc (Sigma), anti-hGH, anti-Neogenin, and goat polyclonal anti-DCC (Santa Cruz Biotechnology) were used. To analyze the forebrain commissure phenotype and the expression of DCC proteins, cryostat sections (16 $\mu \mathrm{m}$ thick) were subjected to immunofluorescence analysis (Islam et al., 2009) with antibodies: rat anti-L1 (Millipore Bioscience Research Reagents) and goat anti-human DCC (Santa Cruz Biotechnology).

Binding assay and determination of dissociation constant for draxinDCC binding. 293T cells were transfected with cDNA encoding chick draxin-AP and empty $A P$ tag vector (Islam et al., 2009) and an alkaline phosphatase assay (Flanagan and Cheng, 2000) using para-

Neogenin
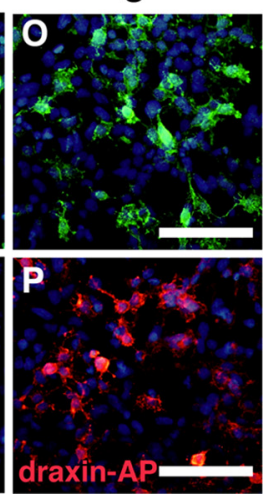

nitrophenylphosphate was used to determine empirically draxin-AP and control-AP concentrations.

Cell overlay assay and other binding experiments were performed as previously described (Keino-Masu et al., 1996; Islam et al., 2009). In the case of draxin binding in the presence of netrin-1, Dcc-transfected 293 cells were first incubated with $12 \mathrm{~nm}$ of netrin-1-his protein (R\&D Systems) for 30 min followed by the incubation of draxin-AP-conditioned medium. Saturation binding curves and Scatchard analyses of draxin-AP binding to DCC were performed, and the dissociation constant $\left(K_{\mathrm{d}}\right)$ value was determined as previously described (Flanagan and Cheng, 2000).

Pull-down assay. Dcc-transfected cells were washed with HBAH buffer [HBSS containing $0.5 \mathrm{mg} / \mathrm{ml}$ bovine serum albumin, $0.1 \%(\mathrm{w} / \mathrm{v})$ sodium azide, and $20 \mathrm{~mm}$ HEPES] and incubated with $1 \mathrm{~nm}$ draxin-AP-conditioned medium for $2 \mathrm{~h}$ at room temperature with occasional gentle stirring. Later, cells were lysed with lysis buffer (50 mM HEPES pH 7.6, $150 \mathrm{~mm} \mathrm{NaCl}, 0.1 \%$ Triton X-100) supplemented with protease inhibitors (1 mM PMSF, $10 \mu \mathrm{g} / \mathrm{ml}$ aprotinin, $10 \mu \mathrm{g} / \mathrm{ml}$ leupeptin). As a negative control, cell lysates from vectortransfected cell, which were incubated with draxin-AP, and Dcc-transfected cells, which were not incubated with draxin-AP, were taken. Lysates were incubated with anti-chick draxin polyclonal antibody (pAb) $(1 \mu \mathrm{g} / \mathrm{ml})$ in immunoprecipitation (IP) buffer (150 mM $\mathrm{NaCl}, 20$ mм Tris-HCl, pH 7.5, 1.5 mm CaCl2, $1.5 \mathrm{~mm} \mathrm{MgCl} 2,0.1 \%$ Triton X-100, $0.1 \%$ CHAPS, $5 \%$ glycerol, and $0.1 \%$ BSA) for $12 \mathrm{~h}$ at $4^{\circ} \mathrm{C}$, and complexes were retained and washed extensively on protein G-Sepharose beads (GE Healthcare). Immunoprecipitated DCC protein was separated by 7.5\% SDS-PAGE and detected by Western blot using anti-DCC antibody.

Concentrated draxin-AP- and DCC-ecto-Myc-His fusion proteinconditioned medium was mixed together and incubated with anti-chick draxin pAb $(1 \mu \mathrm{g} / \mathrm{ml})$ in IP buffer at $4^{\circ} \mathrm{C}$ for $12 \mathrm{~h}$ and then detected by Western blot using mouse monoclonal anti-DCC (Calbiochem). For pull down of draxin-AP by DCC-ecto-Myc-His protein, ProBond resin (Invitrogen) was used. As a negative control, draxin-AP was mixed with BSA. For the pull-down assay of draxin-AP by Robol-ecto-Fc, protein G-Sepharose (GE Healthcare) was used.

Brains from E17 mouse embryos were dissected and lysed in lysis buffer supplemented with protease inhibitors, as described above. The mixtures were kept on ice for $30 \mathrm{~min}$ and centrifuged at 15,000 rpm at $4^{\circ} \mathrm{C}$ for $20 \mathrm{~min}$. Membrane fractions were collected, and lysates were incubated with concentrated draxin-AP-conditioned medium and antichick draxin $\mathrm{mAb}$ for $12 \mathrm{~h}$ at $4^{\circ} \mathrm{C}$. Immunoprecipitates were recovered and analyzed as described above.

Explant cultures. Explants from E17.5 OB and cortex of both wild-type (WT) and Dcc knock-out (KO) littermate mice were dissected as described previously (Shu and Richards, 2001) and cultured in collagen gels for $48 \mathrm{~h}$ in the presence of either draxin-AP- or control-AP-conditioned medium (100 nM) mixed with Neurobasal medium (Invitrogen) supplemented with B27 (Invitrogen), glutamax-I, and penicillin/streptomycin (Invitrogen). After culture, explants were stained with anti-neuronspecific $\beta$-tubulin (Tuj1) antibody (R\&D Systems) in the collagen gels. Photographs were taken using a microscope (Keyence, Biorevo), and the longest neurite length per explant was measured using ImageJ software. Averages were determined from counted maximal lengths of all explants. 
Growth cone collapse and neurite outgrowth assay. Cortical neurons from mouse embryo at E16.5 were dissociated by trypsinization as described previously (Hata et al., 2006) and seeded at low density in 4-well dishes containing coverslips coated with poly-L-Lysine (100 $\mu \mathrm{g} / \mathrm{ml})$ and laminin $(20 \mu \mathrm{g} / \mathrm{ml})$.

For neurite outgrowth assay, neurons were cultured in neurobasal medium (B27, glutamax, penicillin-streptomycin) having equal amounts of either draxin-AP- or control-AP-conditioned medium for $48 \mathrm{~h}$ and were stained with anti-DCC.

For the growth cone-collapse experiment, neurons were seeded in a dish, as above, and cultured in neurobasal medium. After $60 \mathrm{~h}$ of postseeding, neurons were incubated with draxin-AP (100 nM) and control medium alone for $1 \mathrm{~h}$ at $37^{\circ} \mathrm{C}$ to induce collapse. Cultures were fixed later by $4 \%$ paraformaldehyde $/ 10 \%$ sucrose for $1 \mathrm{~h}$. After washing well with PBS, growth cones were stained with phalloidinAlexa 568 (Invitrogen), and images were acquired on a microscope (Model Eclipse E600, Nikon) for scoring the collapse. To calculate the percentage of the collapse, the total number of growth cones was divided by the number of collapsed growth cones. Data were collected from at least two independent experiments and expressed as means \pm SEM. In each independent experiment, at least 40 neurons were counted per group.

Mice. To breed homozygous Dcc mutant embryos, $D c c$ heterozygous mutant mice (Fazeli et al., 1997) were mated. For double-hetero mice analysis, $D c c$ and draxin single-hetero mice were mated. The day of the vaginal plug was designated E0.5. All animal procedures were conducted in accordance with institutional guidelines.

\section{Results}

Netrin-1 receptors bind draxin

We screened several candidate transmembrane proteins by transient expression in 293 cells and measured the binding of draxin-AP (alkaline phosphatase). Surprisingly, the netrin receptors DCC, Neogenin, UNC5s (H1, H2, H3), and DSCAM were found to be promising candidates because draxin-AP was bound to cells expressing those receptors but not to any of the other candidates tested (neuropilin1, FGFR1, EphB1, and Ryk). To examine whether receptor expression and bound draxin-AP colocalize, we assessed the draxin-AP binding and netrin-1 receptors expression in receptor-transfected 293 cells by immunocytochemistry (Fig. $1 A-P)$. We observed that draxin-AP binding completely overlapped with DCC, UNC5s (H1, H2, H3), and DSCAM expression (Fig. $1 C-L$ ) in a manner similar to netrin-1 binding (Fig. $1 A, B$ ), but not with the expression of NCAM (Fig. $1 M, N$ ), a member of the Ig superfamily. We also observed a similar pattern of draxin binding and Neogenin expression (Fig. 1O,P) when draxin binding was performed separately in Neogenin-transfected 293 cells. Thus, draxin binding was restricted to netrin-1 receptor-expressing cells.

Draxin interacts with DCC with high affinity, and its binding domain in DCC is different from the netrin-1 binding domain Among the netrin-1 receptors, only Dcc KO mice phenotype similarity with draxin knockouts (Fazeli et al., 1997; Islam et al.,

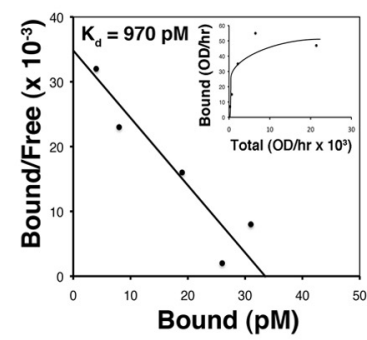

H

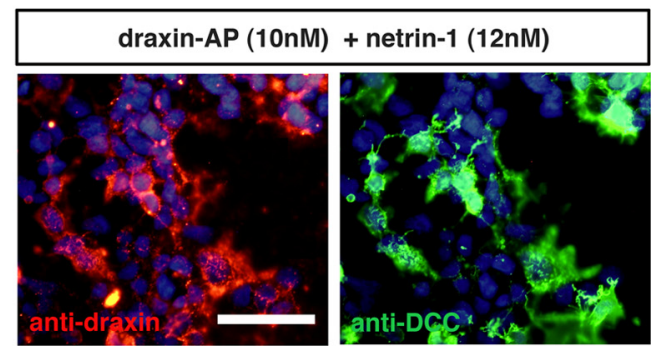

B

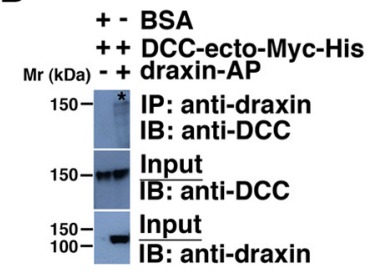

E

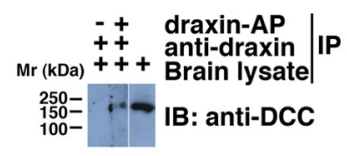

C

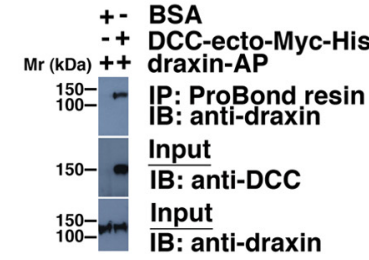

$\mathbf{F}$

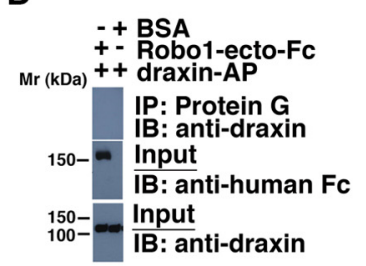

IB: anti-DCC

Figure 2. Draxin interacts with DCC by distinct domain of netrin-1 binding. $A, D c c-$ transfected 293 cells were incubated with or without draxin-AP-conditioned medium, and later cell lysates were subjected to IP assay. DCC protein in cell lysates and immunoprecipitates was detected by anti-DCC antibody. IB, Immunoblot. B, Conditioned medium from Dcc-ecto-Myc-His-transfected 293 列 -AP when both proteins were mixed and subjected to pull-down assay. ProBond resin bound His-tag. D, Draxin-AP was draxin-AP, and IP assay was performed using anti-draxin as an IP antibody. Endogenous DCC protein from E17 mouse brain lysates

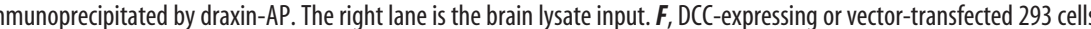

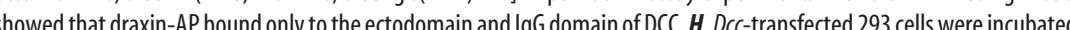
performed to see the bound draxin in the DCC expressing cells. Scale bar, $50 \mu \mathrm{m}$.

2009) led us to investigate the draxin/DCC interaction thoroughly. To test whether draxin interacts directly with DCC, first we performed immunoprecipitation assays. DCC was retained in the complex precipitated by draxin-AP, as detected by immunoblot using an anti-DCC antibody (Fig. $2 A$ ). To determine whether the interaction between DCC and draxin was direct, we performed IP assays using draxin-AP and the soluble DCCectodomain. DCC-ectodomain was specifically immunoprecipitated by draxin-AP (Fig. $2 B$ ). In addition, draxin-AP was pulled down by the DCC-ectodomain (Fig. 2C). The interaction between draxin and DCC appeared specific, because a soluble version of the Robol ectodomain (Robol-ecto-Fc) did not precipitate draxin-AP (Fig. 2D). Moreover, endogenous DCC from brain lysates was precipitated by draxin-AP (Fig. $2 E$ ). The binding affinity of draxin for DCC in transfected cells was determined from the binding curve, and the $K_{\mathrm{d}}$ was 970 pM (Fig. $2 F$ ), higher than that of the DCC-netrin-1 interaction in a similar assay (Keino-Masu et al., 1996). We next sought to identify the draxinbinding domain of DCC by evaluating the binding of draxin to the 


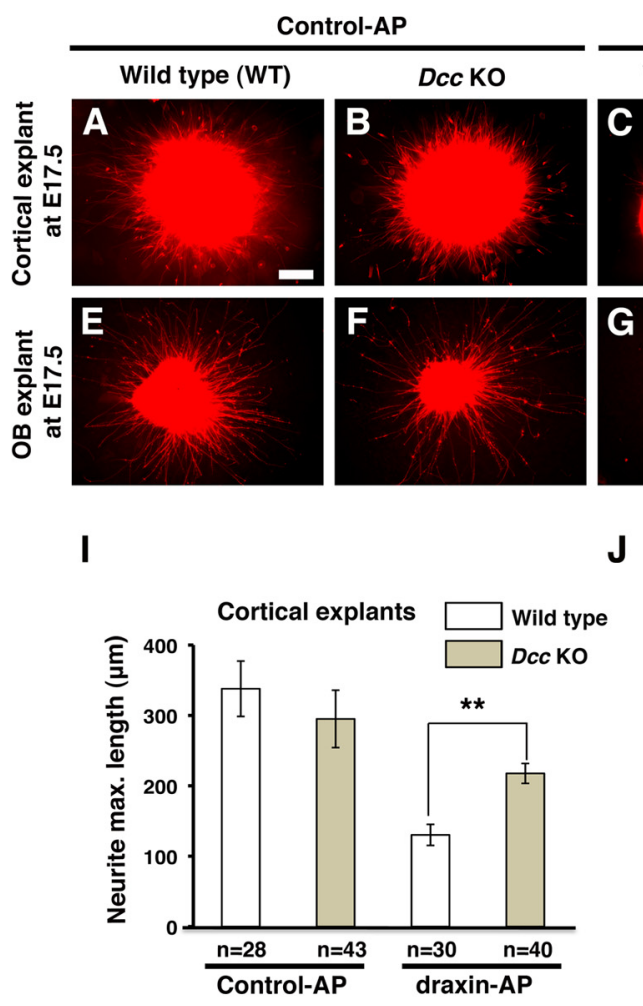

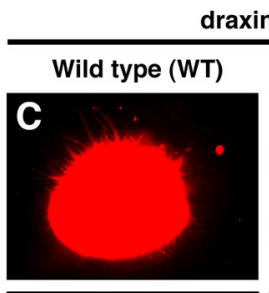

raxin-AP

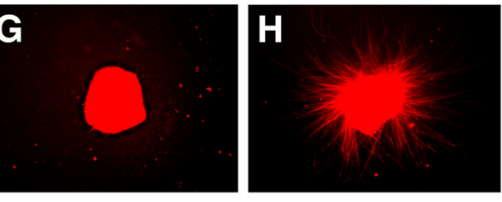

$\mathbf{J}$

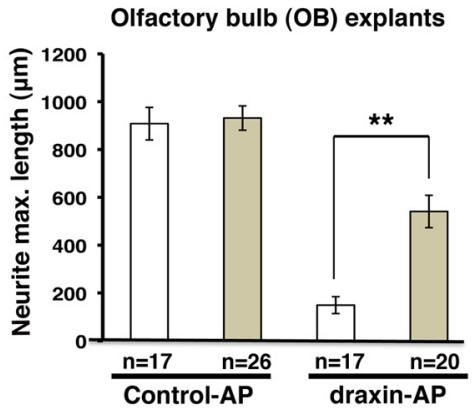

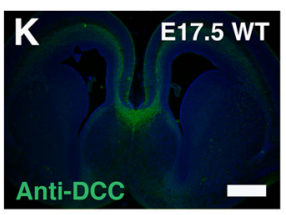
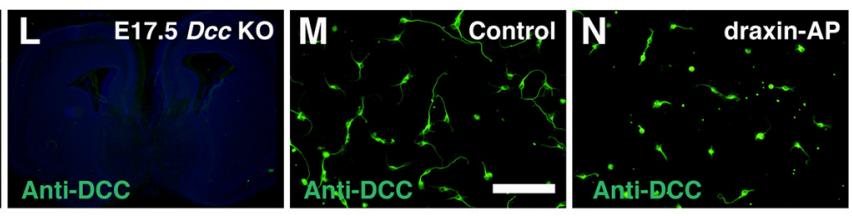

0

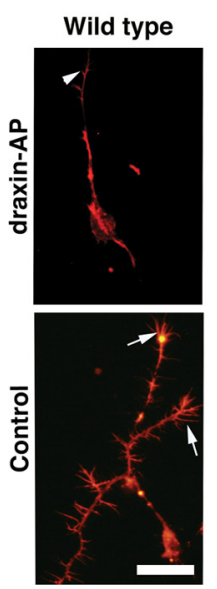

$\mathbf{P}$

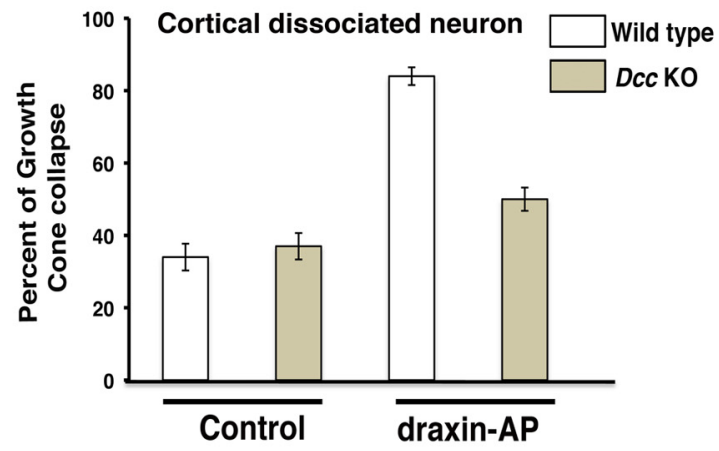

Figure 3. Dcc-deficient neurite outgrowth and growth cone collapse are significantly less affected by draxin. $\boldsymbol{A}-\boldsymbol{H}$, Cortical $(\boldsymbol{A}-\boldsymbol{D})$ and $\mathrm{OB}(\boldsymbol{E}-\boldsymbol{H})$ explants from $E 17.5 \mathrm{WT}(\boldsymbol{A}, \boldsymbol{C}, \boldsymbol{E}, \boldsymbol{G})$ and $\operatorname{DCC} \mathrm{KO}(\boldsymbol{B}, \boldsymbol{D}, \boldsymbol{F}, \boldsymbol{H})$ mice were cultured in collagen gel in the presence of either control-AP-conditioned $(\boldsymbol{A}, \boldsymbol{B}, \boldsymbol{E}, \boldsymbol{F})$ or draxin-AP-conditioned $(\boldsymbol{C}, \boldsymbol{D}, \boldsymbol{G}, \boldsymbol{H})$ medium for $48 \mathrm{~h}$. Neurites were labeled using an antibody to class III $\beta$-tubulin, and results were quantified $(I, J)$ by measuring the maximum neurite length per explant from cortex $(\boldsymbol{A}-\boldsymbol{D})$ and $\mathrm{OB}(\boldsymbol{E}-\boldsymbol{H})$. The total number of explants, $n$ in $\boldsymbol{I}$ and $\boldsymbol{J}$, was derived from two independent experiments, and the average maximum neurite length was determined. Similar results were observed in the other two independent experiments. Neurites of cortical $(I)$ and $O B(J)$ explants from Dcc KO mice were significantly $\left({ }^{* *} p<0.0001\right.$, Student's $t$ test) less inhibited by draxin-AP compared with those from WT mice. $\boldsymbol{K}, \boldsymbol{L}$, Specific DCC immunoreactivity was observed only in the coronal section of E17.5 cortex from WT mouse $(\boldsymbol{K})$ but not from Dcc KO mouse $(\boldsymbol{L})$. Cortical neurons from E16.5 wild-type mice embryos were cultured on poly-L-lysine/laminin-coated dishes either in the presence of control medium-conditioned $(\boldsymbol{M})$ or draxin-AP-conditioned $(\boldsymbol{N})$ medium for $40 \mathrm{~h}$. Neurites were stained with anti-DCC antibody and inhibited markedly by draxin-AP. Growth cones of E16.5 cortical neurons from both WT and Dcc KO littermate mice were treated with either medium alone or draxin-AP $100 \mathrm{~nm}$ for $1 \mathrm{~h}$ at $37^{\circ} \mathrm{C}$ to induce the collapse and were stained with phalloidin-Al 568 to visualize the growth cones. Representative pictures were soluble form of fragmented DCC proteins by pull-down assay. Whereas netrin-1 binds the fibronectin-type-III-like region of DCC (Geisbrecht et al., 2003; Kruger et al., 2004), draxin preferentially bound the IgG (1-4) domain of DCC, rather than the FN (1-6) domain (Fig. 2G). We then assessed draxin binding in Dcc-transfected 293 cells in presence of netrin-1 by immunocytochemistry. We found that draxin binding to DCC was not inhibited (Fig. $2 H$ ), indicating that draxin and netrin-1 binding domain in DCC are different. These biochemical data confirm the specificity of the interaction between draxin and DCC.

DCC is required for neurite outgrowth inhibition and growth cone collapse by draxin

DCC was reported to be expressed on cortical and OB axons (Gad et al., 1997; Shu et al., 2000), and both Dcc and draxin KO mice show severe malformation of forebrain commissures (Islam et al., 2009; Fazeli et al., 1997). Also, we recently reported that draxin repelled $\mathrm{OB}$ axons in vitro and lateral olfactory tract (LOT) projection in draxin $^{-1-}$ mice was normal, though slightly defasciculated (Ahmed et al., 2010). To determine whether the interaction of draxin with DCC is functionally important, we tested the ability of Dcc-deficient cortical and OB axons to respond to draxin in vitro. Explants from E17.5 cortex and OB of Dcc KO and WT littermate mice were cultured in the presence of either draxin-AP- or control-APconditioned medium. Robust neurite outgrowth from both cortical and $\mathrm{OB}$ explants of WT mice was observed in control-AP-conditioned medium (Fig. $3 A, E$ ), and a similar degree of outgrowth was observed from explants of Dcc knock-out mice in the presence of control-AP (Fig. $3 B, F)$. Draxin-AP significantly inhibited neurite outgrowth from cortical and $\mathrm{OB}$ explants of WT mice to 38 and 16\%, respectively, of control levels (Fig. 3C,G,I,J), whereas neurite growth in $D c c$-deficient cortical and $\mathrm{OB}$ explants was inhibited to 64 and $59 \%$ of control levels, respectively (Fig. $3 D, H, I, J)$. These findings demonstrate that DCC is substantially required for draxin to inhibit neurite outgrowth.

$\longleftarrow$

shown in $\mathbf{0}$, and quantified results were shown in $\boldsymbol{P}$. Data were obtained from two independent experiments, and growth cones of 40 neurons were counted in each group on average per experiment. Arrows and arrowhead in $\mathbf{0}$ indicate the intact growth cone and collapsed cone, respectively. Scale bars: $\boldsymbol{A}-\boldsymbol{H}, 200 \mu \mathrm{m} ; \boldsymbol{K}, \boldsymbol{L}, 100 \mu \mathrm{m} ; \boldsymbol{M}-\mathbf{0}, 50 \mu \mathrm{m}$. Error bar indicates mean $\pm S E$ 
Draxin not only inhibits neurite outgrowth but also induces growth cone collapse when added acutely and uniformly to growth cones in culture (Islam et al., 2009). We therefore investigated whether DCC is required for this acute response. For this assay, we used E16.5 mouse cortical neurons, which express DCC and were inhibited by draxin (Fig. $3 M, N$ ). The specificity of the antibody was confirmed when DCC immunoreactivity was observed in the sections of WT but not of Dcc KO mice (Fig. $3 K, L$ ). We found that growth cones in this culture system had a high baseline level of collapse ( $\sim 35 \%)$, which was further increased (to $\sim 85 \%$ ) when treated with draxin-AP (Fig. 3O,P). This increase was markedly reduced (to $\sim 50 \%$ ) in cortical neurons derived from Dcc KO mice (Fig. 3P).

\section{Dcc/draxin double-hetero embryos exhibit severe defects in CC}

It was reported earlier that both $D c c$ and draxin single-knock-out mice showed severe defects in forebrain commissure formation and mild disruption or defasciculation of LOT projection (Fazeli et al., 1997; Islam et al., 2009; Kawasaki et al., 2006; Ahmed et al., 2010). To evaluate whether genetic interaction between $D c c$ and draxin is functionally important for the formation of forebrain commissures and LOT, we mated draxin and Dcc single-hetero mice. Mating of these mice generated babies with the following four different genotypes: $\operatorname{draxin}^{+/+} \mathrm{Dcc}^{+/+}$(WT); $\operatorname{draxin}^{+/-}$ $\mathrm{Dcc}^{+/+}$; $\operatorname{draxin}^{+1-} \mathrm{Dcc}{ }^{+/-}$; and $\operatorname{draxin}^{+/+} \mathrm{Dcc}{ }^{+/-}$. First, we compared the forebrain commissure phenotypes in babies belonging to draxin ${ }^{+/-} \mathrm{Dcc}^{+/+}$, draxin $^{+/-} \mathrm{Dcc}^{+/-}$, and draxin ${ }^{+/+}$ $\mathrm{Dcc}^{+/-}$mice. We observed notable variation in severity in CC defect but not in anterior and hippocampal commissure defect in single- and double-hetero mice. We classified the phenotype into two categories depending on the severity in CC defects. The CC axons in mice in the strong phenotype group failed to cross the midline and projected ventrally (Fig. 4C), while mice in the weak group showed partial misprojection near the midline (Fig. $4 B$ ). Whereas the frequencies of strong phenotypes observed in draxin and Dcc single-hetero mice were 0 and $11 \%$, respectively, doublehetero draxin/Dcc mice showed a remarkably higher (62\%) frequency of strong phenotype (Fig. 4D). Next, we checked whether LOT formed and reached normally to the telencephalon of these analyzed mice. We found that LOT formation was not disrupted and reached normally in all the single- and double-hetero mice (data not shown). This was not unexpected since LOT was normally formed in both draxin and Dcc single-knock-out mice despite mild defasciculation (Kawasaki et al., 2006; Ahmed et al., 2010). In these double-hetero mice, other guidance molecules and their receptors having redundant function to draxin might compensate the role of draxin in LOT formation. Overall, these data indicated that the aggravated CC defect in double-hetero mice could be a result of genetic interaction between draxin and $D c c$ genes.

\section{Discussion}

We have shown here that draxin binds multiple netrin-1 receptors by cell overlay assay; its interaction with DCC is especially functionally important since Dcc-lacking cortical and olfactory neurites were substantially less sensitive to draxin in vitro. Furthermore, the degree of growth cone collapse of Dcc KO cortical neurites in response to draxin was significantly lower than that of wild-type neurons. In vivo, draxin/Dcc double-hetero mice showed severe defects in CC compared with either of the single heteros. Together, these data indicate that DCC is a functionally important receptor for draxin-mediated neurite outgrowth inhibition and guidance.

Because DCC is a receptor for netrin-1, it will be of interest to determine how DCC can mediate signaling cascades for two such unrelated ligands, especially since we previously showed that spinal commissural axons can simultaneously respond to netrin-1 with outgrowth and to draxin with repulsion. The answer may lie partly in the structure of DCC, which in principle could bind different ligands through different binding domains. In fact, our data show that the draxin binding site on DCC is different from that of netrin-1. A growing body of literature indicates that the interaction of DCC with netrin-1 can lead to switching on or off of diverse intracellular processes (Ming et.al., 1997; Li et al., 2004; Moore et al., 2008). There is a possibility that the downstream events that result from the DCC-draxin interaction are complementary to those triggered by DCC-netrin-1 binding. In addition, the DCC-draxin interaction might recruit other molecules as coreceptors; for example, DCC-netrin-1 can recruit UNC5 family members to mediate a repulsive action of netrin-1 (Hong et al., 1999). The probability of this notion is high since draxin also binds with UNC5s. Also, the possibility of a coreceptor that can also bind draxin is suggested by the finding that the inhibitory effect of draxin was not completely abolished by removal of DCC; this would parallel the situation with netrin, since UNC5 family members can also bind netrin and by themselves mediate repulsion, though a DCC-UNC5 coreceptor is a more potent mediator of repulsion (Keleman and Dickson, 2001).

While a conserved role for DCC in mediating netrin responses, both attractive and repulsive, has been well documented in multiple species, in Caenorhabditis elegans, the DCC homolog UNC-40 was also shown to collaborate with the Slit receptor Sax-3/Robo in mediating repulsion in response to SLT-1/Slit (Yu 
et al., 2002); however, DCC family members do not appear to bind Slit proteins, and this effect appears instead to reflect a direct interaction of the receptors UNC-40/DCC with Sax-3 (Yu et al., 2002). Our results extend the principle of DCC involvement in mediating the effects of distinct ligands, but indicate that it can also involve direct binding of the ligand, as we show here for draxin. Since commissural axons can be simultaneously stimulated to extend by netrin-1 and repelled by draxin, it will be of particular interest to determine how DCC can be involved at the same time in mediating these apparently opposite functions. The identification of DCC as a receptor for draxin is an important step to further elucidation of downstream components of DCCdraxin signaling.

\section{References}

Ahmed G, Shinmyo Y, Naser IB, Hossain M, Song X, Tanaka H (2010) Olfactory bulb axonal outgrowth is inhibited by draxin. Biochem Biophys Res Commun 398:730-734.

Dickson BJ (2002) Molecular mechanisms of axon guidance. Science 298:1959-1964.

Fazeli A, Dickinson SL, Hermiston ML, Tighe RV, Steen RG, Small CG, Stoeckli ET, Keino-Masu K, Masu M, Rayburn H, Simons J, Bronson RT, Gordon JI, Tessier-Lavigne M, Weinberg RA (1997) Phenotype of mice lacking functional deleted in colorectal cancer (Dcc) gene. Nature 386:796-804.

Flanagan JG, Cheng HJ (2000) Alkaline phosphatase fusion proteins for molecular characterization and cloning of receptors and their ligands. Methods Enzymol 327:198-210.

Gad JM, Keeling SL, Wilks AF, Tan SS, Cooper HM (1997) The expression patterns of guidance receptors, DCC and Neogenin, are spatially and temporally distinct throughout mouse embryogenesis. Dev Biol 192:258-273.

Geisbrecht BV, Dowd KA, Barfield RW, Longo PA, Leahy DJ (2003) Netrin binds discrete subdomains of DCC and UNC5 and mediates interactions between DCC and heparin. J Biol Chem 278:32561-32568.

Hata K, Fujitani M, Yasuda Y, Doya H, Saito T, Yamagishi S, Mueller BK, Yamashita T (2006) RGMa inhibition promotes axonal growth and recovery after spinal cord injury. J Cell Biol 173:47-58.

Hong K, Hinck L, Nishiyama M, Poo MM, Tessier-Lavigne M, Stein E (1999) A ligand gated association between cytoplasmic domains of UNC5 and
DCC family receptors converts netrin-induced growth cone attraction to repulsion. Cell 97:927-941.

Islam SM, Shinmyo Y, Okafuji T, Su Y, Naser IB, Ahmed G, Zhang S, Chen S, Ohta K, Kiyonari H, Abe T, Tanaka S, Nishinakamura R, Terashima T, Kitamura T, Tanaka H (2009) Draxin, a repulsive guidance protein for spinal cord and forebrain commissures. Science 323:388-393.

Kawasaki T, Ito K, Hirata T (2006) Netrin 1 regulates ventral tangential migration of guidepost neurons in the lateral olfactory tract. Development 133:845-853.

Keino-Masu K, Masu M, Hinck L, Leonardo ED, Chan SS, Culotti JG, TessierLavigne M (1996) Deleted in colorectal cancer (DCC) encodes a netrin receptor. Cell 87:175-185.

Keleman K, Dickson BJ (2001) Short and long-range repulsion by the Drosophila Unc5 netrin receptor. Neuron 32:605-617.

Kruger RP, Lee J, Li W, Guan KL (2004) Mapping netrin receptor binding reveals domains of Unc5 regulating its tyrosine phosphorylation. J Neurosci 24:10826-10834.

Li W, Lee J, Vikis HG, Lee SH, Liu G, Aurandt J, Shen TL, Fearon ER, Guan JL, Han M, Rao Y, Hong K, Guan KL (2004) Activation of FAKand Src are receptor-proximal events required for netrin signaling. Nat Neurosci 7:1213-1221.

Ming GL, Song HJ, Berninger B, Holt CE, Tessier-Lavigne M, Poo MM (1997) cAMP-dependent growth cone guidance by netrin-1. Neuron 19:1225-1235.

Moore SW, Correia JP, Lai Wing Sun K, Pool M, Fournier AE, Kennedy TE (2008) (2008) Rho inhibition recruits DCC to the neuronal plasma membrane and enhances axon chemoattraction to netrin 1. Development 135:2855-2864.

Shu T, Richards LJ (2001) Cortical axon guidance by the glial wedge during the development of the corpus callosum J Neurosci 21:2749-2758.

Shu T, Valentino KM, Seaman C, Cooper HM, Richards LJ (2000) Expression of the netrin-1 receptor, deleted in colorectal cancer (DCC), is largely confined to projecting neurons in the developing forebrain J Comp Neurol 416:201-212.

Tessier-Lavigne M, Goodman CS (1996) The molecular biology of axon guidance. Science 274:1123-1133.

Yu TW, Hao JC, Lim W, Tessier-Lavigne M, Bargmann CI (2002) Shared receptors in axon guidance: Sax-3/Robo signals via UNC-34/enabled and a netrin-independent UNC-40/DCC function. Nat Neurosci 5:11471154.

Zou Y, Lyuksyutova AI (2007) Morphogens as conserved axon guidance cues. Curr Opin Neurobiol 17:22-28. 